\title{
The Effect of $N^{6}, O^{2}$-Dibutyryl Adenosine-3',5'-cyclic Monophosphate on Morphogenesis in Mucorales
}

\author{
By B. E. JONES* AND J. D. BU'LOCK \\ Weizmann Microbial Chemistry Laboratory, Department of Chemistry, \\ University of Manchester, Manchester $M \mathrm{I} 3{ }_{9} P L$
}

(Received 24 June 1977)

\begin{abstract}
The effect of adding dibutyryl cyclic AMP to five species of Mucorales was tested. With Mucor hiemalis and $M$. mucedo a rapid halt of apical extension and a slower rate of resumed growth were observed together with marked morphological changes, including hyphal dilation, apical swelling, septation and multiple branching. Sporangiospore development, and the mating reaction in $M$. mucedo were both inhibited; carotene pigmentation was increased. Phycomyces blakesleeanus, Blakeslea trispora and Zygorhynchus moelleri were insensitive to dibutyryl cyclic AMP.
\end{abstract}

\section{INTRODUCTION}

Sexual reproduction in heterothallic Mucorales follows a sequence of well-defined morphogenetic events controlled by the hormone trisporic acid, whose synthesis is in turn dependent on, inter alia, nutritional regulation (Gooday, 1973; Bu'Lock, 1976; Bu'Lock, Jones \& Winskill, 1976). The involvement of cyclic AMP as an intracellular mediator for at least some of these events is possible a priori. Cyclic AMP levels are elevated during sexual morphogenesis in Mucor mucedo (Bu'Lock et al., 1976) and cyclic AMP is apparently involved in the phototropic response of sporangiophores of Phycomyces blakesleeanus (Cohen, 1974), a process which may be related to the zygotropic response during sexual differentiation.

To test the effects of cyclic AMP on some heterothallic and homothallic species of Mucorales, we chose the lipophilic dibutyryl derivative because of its better membrane permeability and greater resistance to cyclic AMP phosphodiesterase. An abstract of some of this work has been published in Phycomyces Meeting 1976, Cold Spring Harbor Laboratory.

\section{METHODS}

Organisms and media. Mucor mucedo (L. ex Fres.) Brefeld, strains z46 (+) and z43 (-), a mating pair obtained from Dr G. W. Gooday (University of Aberdeen), was maintained at $4{ }^{\circ} \mathrm{C}$ after growth at $20^{\circ} \mathrm{C}$ on potato dextrose agar (Oxoid). Mucor hiemalis Wehmer, strains NRRL246I (+) and NRRL2462 (-), a mating pair obtained from Dr C. W. Hesseltine (Peoria, U.S.A.), was maintained on potato dextrose agar or $2 \%(\mathrm{w} / \mathrm{v})$ commercial malt extract agar at $4{ }^{\circ} \mathrm{C}$ after growth at $26^{\circ} \mathrm{C}$. The azygosporic (az) strains of $M$. hiemalis, 795-I, 795-4 and 795-5, were a gift from Professor W. L. Gauger (Nebraska, U.S.A.). Each was a single vegetative spore isolate from (az) strain 792-3, itself a single vegetative spore isolate from (az) strain I06B (Gauger, 1966, 1975). The azygosporic condition cannot be maintained by mycelial transfer for any length of time. To retain the 'unique' nature of the strains, spores were suspended in $5 \%(\mathrm{w} / \mathrm{v}) \mathrm{dried}$ skimmed milk (Marvel; Cadbury Ltd, Bournville, Birmingham) solution and maintained on silica gel (Onions, 197I). Phycomyces blakesleeanus Burgeff, strains NRRLI554 (+) and NRRLI555(-), a mating pair supplied by

* Present address: Department of Microbiology, University of Aberdeen, Marischal College, Aberdeen AB9 IAS. 
Professor Gauger, was maintained on $2 \%$ commercial malt extract agar at $4{ }^{\circ} \mathrm{C}$ after growth at $26^{\circ} \mathrm{C}$. Zygorhynchus moelleri Vuillemin, a homothallic species, was a gift from Dr G. H. Banbury (University of Durham) and was maintained on potato dextrose agar at $4{ }^{\circ} \mathrm{C}$ after growth at $26^{\circ} \mathrm{C}$. Blakeslea trispora Thaxter, strains MP2 $(+)$ and MMI (-), a mating pair used in this laboratory, had been vegetatively transferred for some years from NRRL2875 (+) and NRRL2896(-). The strains were maintained on glucose/ asparagine/salts medium (Bu'Lock \& Winstanley, 1971).

Chemicals. $N^{\mathrm{b}}, O^{2}$-Dibutyryl adenosine-3', $5^{\prime}$-cyclic monophosphate, monosodium salt (Boehringer) was dissolved in distilled water for growth and morphology experiments; for hyphal extension experiments, it was dissolved in $2 \%(\mathrm{w} / \mathrm{v})$ aqueous commercial malt extract solution $(\mathrm{I} \cdot 0 \mathrm{ml})$ so that $\mathrm{I}$ o $\mu \mathrm{l}$ contained $0.2 \mu \mathrm{mol}$ dibutyryl cyclic AMP. Theophylline (1,3-dimethylxanthine) at $1.960 \mathrm{mg} \mathrm{ml}^{-1}$ (10.9 mM) and I $9.60 \mathrm{mg} \mathrm{ml}^{-1}$ (109 mM), and caffeine (1,3,7-trimethylxanthine) at $4.066 \mathrm{mg} \mathrm{ml}^{-1}(20.9 \mathrm{mM})$ were in aqueous solution.

Effect of dibutyryl cyclic AMP on growth and morphology. Fungi were grown from a single inoculation at the periphery of $9 \mathrm{~cm}$ diameter Petri dishes containing $20 \mathrm{ml}$ commercial malt glucose agar (all $2 \%, \mathrm{w} / \mathrm{v}$ ) for $M$. mucedo and Io $\mathrm{ml}$ potato dextrose agar (Oxoid) for other species. Agar plates of (az) strains of $M$. hiemalis were inoculated by transferring a single nodule from the silica gel stock culture. The plates were incubated until mycelium covered between one-half and one-third of the surface, usually after 48 to $72 \mathrm{~h}$. By means of a cork borer, $5 \mathrm{~mm}$ diameter wells were cut in the agar just in front of the colony margin and filled with $30 \mu \mathrm{l}$ test solution ( $50 \mu \mathrm{l}$ for $B$. trispora and P. blakesleeanus). Empty wells and wells containing $30 \mu 1$ water served as controls.

Photographs were taken 20 to $24 \mathrm{~h}$ after the administration of dibutyryl cyclic AMP to wells in Petri dish cultures by applying a drop of saline and adding a coverslip. The cultures were observed using a Zeiss microscope incorporating a $64 / 44$ (green) sequence of barrier filters and a camera attachment with Kodak Panatomic-X film.

Effect of dibutyryl cyclic AMP on apical growth. Petri dishes containing potato dextrose agar (Io $\mathrm{ml}$ ) were inoculated centrally with either (+) or (-) M. hiemalis and the agar was covered with a $7 \mathrm{~cm}$ disc of sterile polypropylene film (Sterilin, Richmond, Surrey) which is permeable to oxygen. The cultures were incubated in the dark for 48 to $72 \mathrm{~h}$. By using a scalpel, the agar, mycelium and polypropylene film were cut into eight sectors and left for $\mathrm{I} h$ to recover. A sector was mounted on a microscope slide which was placed on a Zeiss microscope (model RA38) stage and the culture was allowed to equilibrate at the ambient temperature for $10 \mathrm{~min}$. By means of a $\times 30$ objective and a $\times$ Io eyepiece incorporating a scale, a leading hypha on the agar surface was selected, aligned with the scale and growth was noted at I min intervals for about Io min. If the growth rate was steady, the objective was swung away, the polypropylene film peeled back and dibutyryl cyclic AMP solution or $2 \%$ malt extract solution (control) was delivered directly to the illuminated area. The polypropylene film and the objective were replaced and observations were continued for a total of about 60 min.

Effect of dibutyryl cyclic AMP on mating of M. mucedo. Petri dishes containing malt glucose agar (20 ml) were inoculated with parallel streaks of $(+)$ and $(-) M$. mucedo and incubated for $96 \mathrm{~h}$ at $20^{\circ} \mathrm{C}$. The colonies were then in diffusion contact and the first zygophores had appeared. From the centre of the mating zone a $5 \mathrm{~mm}$ diameter plug of agar was removed and the well was filled with $50 \mu 1$ dibutyryl cyclic AMP (I $\mu$ mol) solution. The plates were re-incubated and examined after 24 and $56 \mathrm{~h}$ when mating success was scored by counting the number of zygospores in successive microscope (Reichert Stereoscan) fields of view $(3.6 \mathrm{~mm}$ diam.) on either side of the well.

\section{RESULTS}

\section{Effect of dibutyryl cyclic AMP on growth and morphology}

In preliminary experiments using $(+)$ and $(-) M$. mucedo as test species and wells containing I $\mu \mathrm{mol}$ or $0.5 \mu \mathrm{mol}$ dibutyryl cyclic AMP (50 $\mu \mathrm{l})$, growth inhibition was apparent after $24 \mathrm{~h}$. Microscopic examination showed a change in morphology, the demarcation line being very distinct. Hyphal branching was reduced and the hyphae were thicker and had swollen tips. Carotene accumulation was visibly higher than in controls. After $24 \mathrm{~h}$, the attenuated growth became mainly sub-surface, emerging from the agar surface in isolated patches of thin feathery hyphae. Normal sporangiophore production was inhibited, and only a few thick, stunted aerial structures formed. Effects on the $(-)$ strain were generally less severe. The zone of affected mycelium extended at least $\mathrm{x} \cdot 5 \mathrm{~cm}$ from the edge of the well by $24 \mathrm{~h}$, and allowing for diffusion of the dibutyryl cyclic AMP through the agar it is evident that very low concentrations of the nucleotide are sufficient to achieve these effects. 

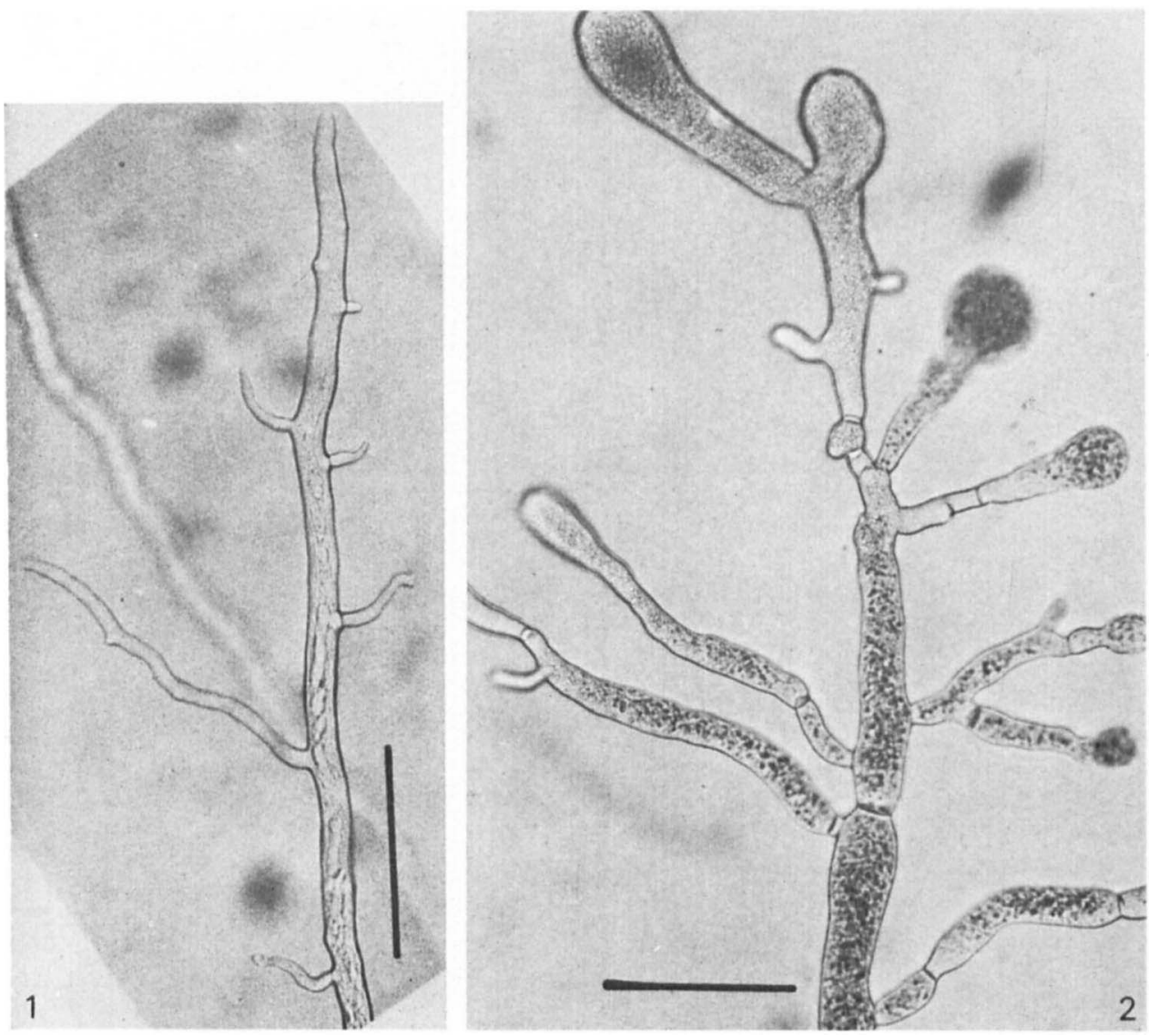

Bar markers represent $100 \mu \mathrm{m}$.

Fig. I. Normal hypha of $M$. hiemalis 795-5.

Fig. 2. Hypha of $M$. hiemalis $795^{-1}$ after the addition of $0.3 \mu \mathrm{mol}$ dibutyryl cyclic AMP.

Similar results were obtained using cyclic AMP, although at higher concentrations (unpublished results).

Using shallower and more transparent potato dextrose agar to aid microscopy, $(+)$ and (-) M. hiemalis and the (az) strains responded in the same manner towards as little as 0.09 to $0 \cdot 1 \mu \mathrm{mol}$ dibutyryl cyclic AMP, showing strong growth inhibition and profound changes in morphology. Generally the $(+)$ and (az) strains were more affected than the $(-)$ strain. The observed morphological changes included multiseptate and swollen hyphae with bulbous or club-shaped tips (Fig. 2). Lateral branching of hyphae was reduced and septa often divided the swollen hyphae into 'strings' of segmented cells (Fig. 3). Abnormal multiple branches emerged from established but swollen hyphal tips (Figs 4 and 5) and there was some lysis of both new and established hyphal apices (Figs 5 and 6). The controls had hyphae that were aseptate, not dilated and without the densely granular appearance of hyphae affected by dibutyryl cyclic AMP (Fig. I). Lateral branching was normally subapical and regular, and hyphal tips were not swollen. In hyphae affected by dibutyryl cyclic AMP, lipid droplets pigmented with carotene were visibly more numerous than usual. Aerial hyphae were slower to develop and much reduced in quantity, but by $44 \mathrm{~h}$ the established surface mycelium had produced some unusual arborescent aerial structures. Azygospores were smoother than usual, and in the case of (az) strain 795-I were formed only beyond a $2 \mathrm{~mm}$ zone around the well.

Neither (+) and (-) B. trispora nor (+) and (-) P. blakesleeanus were affected by up to 
B. E. JONES AND J. D. BU'LOCK
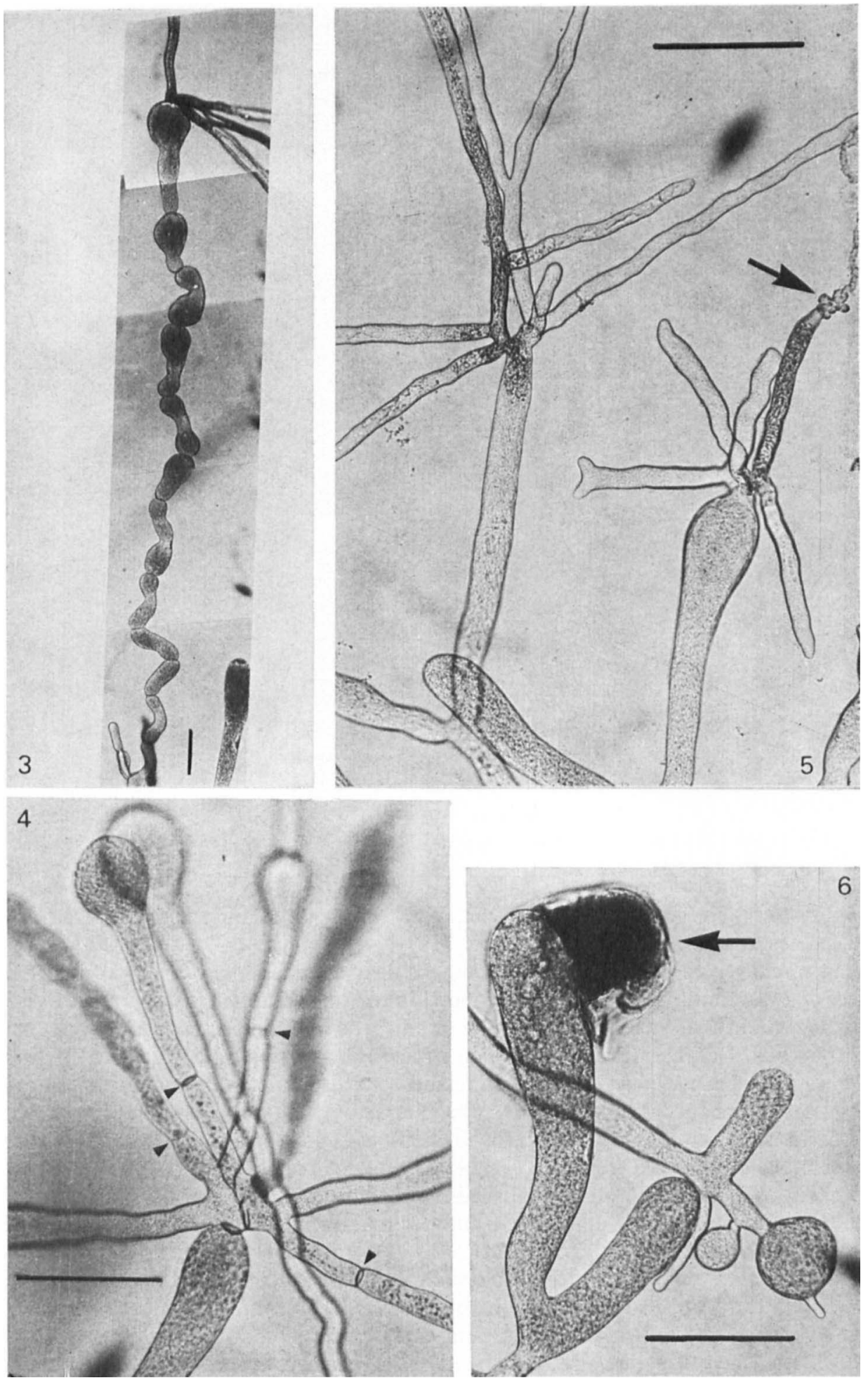


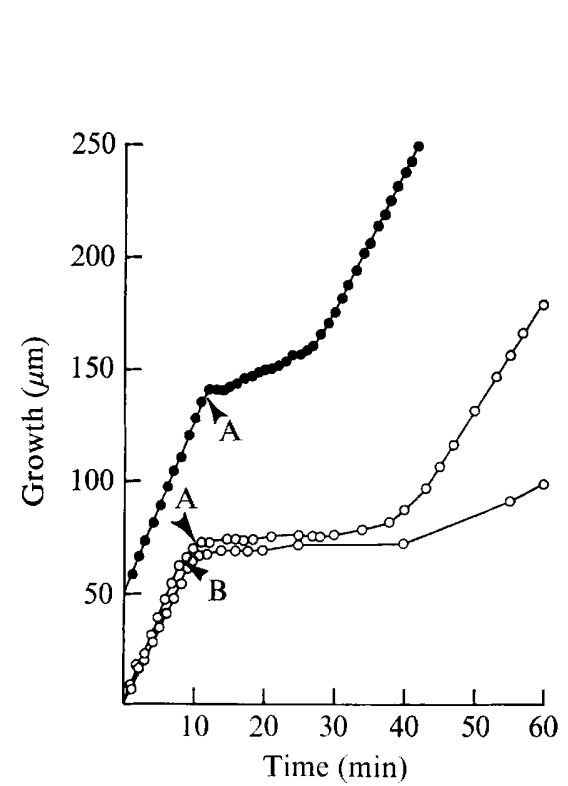

Fig. 7

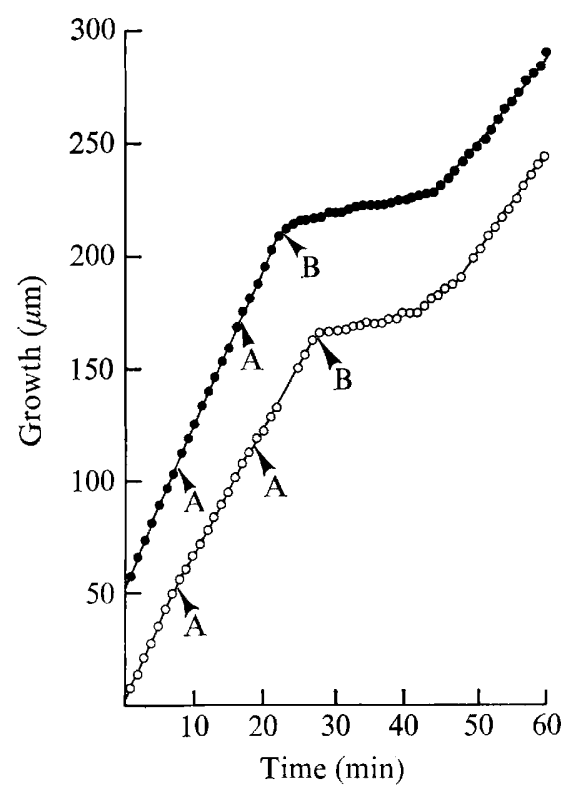

Fig. 8

Fig. 7. Effect of dibutyryl cyclic AMP on the growth of individual hyphae of $(+) M$. hiemalis $(0)$ and $(-) M$. hiemalis $(\bullet):$ A, $0.2 \mu \mathrm{mol}$ dibutyryl cyclic AMP (Io $\mu 1)$ added; B, $0.4 \mu \mathrm{mol}$ dibutyryl cyclic AMP $(20 \mu 1)$ added.

Fig. 8. Effect of successive additions of $2 \%$ malt and dibutyryl cyclic AMP on apical extension of individual hyphae of $(+) M$. hiemalis $(0)$ and $(-) M$. hiemalis $(\bullet):$ A, $2 \%$ malt (Io $\mu \mathrm{l}$ ) added; B, $0 \cdot 2 \mu$ mol dibutyryl cyclic AMP (Io $\mu$ l) added.

$0.4 \mu \mathrm{mol}$ dibutyryl cyclic AMP under the same conditions. The homothallic species $Z$. moelleri did not show any visible effect within $20 \mathrm{~h}$ with up to $12.4 \mu \mathrm{mol}$ dibutyryl cyclic AMP, and normal zygospores were formed.

Theophylline and caffeine inhibit cyclic AMP phosphodiesterase (EC 3. I .4 I7) and can lift the glucose repression of sporulation of Saccharomyces cerevisiae, probably by allowing increased intracellular levels of cyclic AMP (Tsuboi \& Yanagishima, I973). However, these substances had no effect on the growth and morphology of $Z$. moelleri or any of the strains of $M$. hiemalis.

When cycloheximide, an inhibitor of protein synthesis, was administered together with dibutyryl cyclic AMP, some of the effects of the nucleotide were reduced or abolished. However, care must be exercised in interpreting these results because cycloheximide itself causes some growth inhibition. With (+) M. hiemalis the morphological effects of $0 \cdot \mathrm{I} \mu \mathrm{mol}$ dibutyryl cyclic AMP could be completely abolished by $\mathrm{I} \cdot 5 \mu \mathrm{g}$ cycloheximide. This concentration of cycloheximide only reduced the effect of $0.2 \mu \mathrm{mol}$ dibutyryl cyclic AMP;

Bar markers represent $100 \mu \mathrm{m}$.

Fig. 3. Segmented hypha of $M$. hiemalis $795-4$ after the addition of $0.6 \mu \mathrm{mol}$ dibutyryl cyclic AMP. Note that the lipid has occasionally coalesced into larger droplets.

Fig. 4. Mucor hiemalis 795-I with multiple branching from a former hyphal tip after the addition of $0.3 \mu \mathrm{mol}$ dibutyryl cyclic AMP. Note the club-shaped tip and occasional septa (arrowed).

Fig. 5. Mucor hiemalis 795-5 after the addition of $0.6 \mu \mathrm{mol}$ dibutyryl cyclic AMP. Note the multiple branching, granular cytoplasm, apical lysis and extruded cytoplasm (arrowed).

Fig. 6. Mucor hiemalis 795-5 with swollen hyphal tips resulting from the addition of $0.6 \mu$ mol dibutyryl cyclic AMP. Note the dense granular cytoplasm and large lipid droplets. The dense appendage (arrowed) appears to be the result of leakage of intracellular material. 


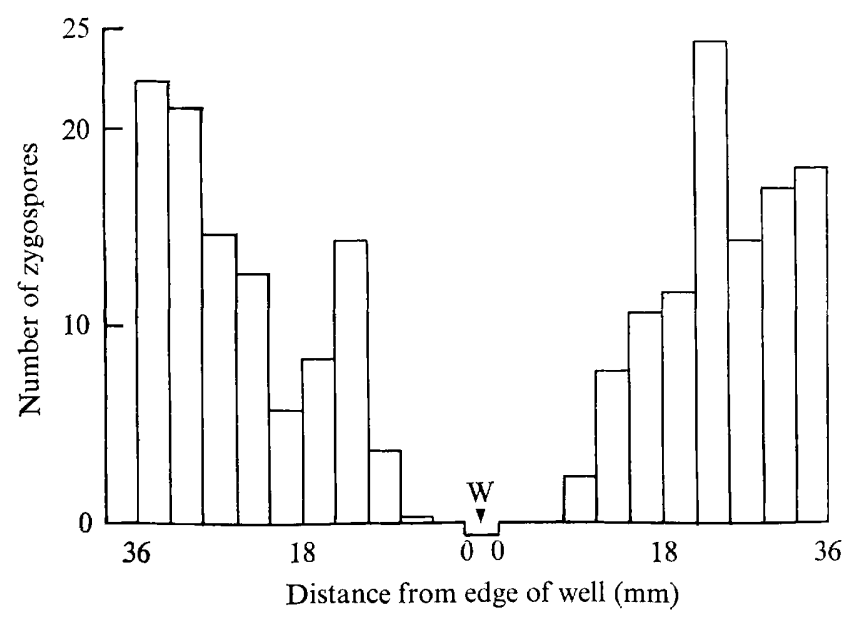

Fig. 9. Histogram showing the number of zygospores in successive microscope fields of view $56 \mathrm{~h}$ after the addition of I $\mu$ mol dibutyryl cyclic AMP to the well (w). The results are the average for three plates.

whilst there was some hyphal dilation, there were no swollen apices, septa or increased carotenoid. With (-) M. hiemalis a higher dose $(4.5 \mu \mathrm{g})$ of cycloheximide was required to eliminate the morphological effects of $0.1 \mu \mathrm{mol}$ dibutyryl cyclic AMP, although growth inhibition and carotenoid accumulation persisted; with $3 \mu \mathrm{g}$ cycloheximide some septation and multiple branching was manifest, but there were no swollen hyphal tips. The effects of $0.2 \mu \mathrm{mol}$ dibutyryl cyclic AMP were not abolished by $4.5 \mu \mathrm{g}$ cycloheximide.

\section{Effect of dibutyryl cyclic AMP on apical growth}

Adding dibutyryl cyclic AMP had a rapid effect on hyphal extension of both $(+)$ and $(-)$ $M$. hiemalis (Figs 7 and 8), but similar additions of $2 \%$ malt solution had little or no effect (Fig. 8). Hyphal growth rates for the (+) strain were typically about $7.0 \mu \mathrm{m} \mathrm{min}^{-1}$, the (-) strain growing slightly faster at about $7.7 \mu \mathrm{m} \mathrm{min}{ }^{-1}$. The addition of malt served only to reduce these rates to about $6.0 \mu \mathrm{m} \mathrm{min}^{-1}$ and $7.0 \mu \mathrm{m} \mathrm{min}^{-1}$ respectively, and lateral branching continued normally. The addition of $0.2 \mu \mathrm{mol}$ dibutyryl cyclic AMP to $(+) M$. hiemalis caused, within seconds, a complete halt of apical growth which lasted about $20 \mathrm{~min}$. During this phase, cytoplasmic streaming was visibly disorganized and large pigmented lipid droplets appeared. The tip swelled and ultimately several branches emerged more or less simultaneously and growth recommenced, but the individual branches grew at a slower rate than before. Meanwhile the established hyphae became swollen and septate. Higher doses of dibutyryl cyclic AMP extended the period of inhibition and resulted in a slower rate of growth when it recommenced (Fig. 7). After adding $0.6 \mu \mathrm{mol}$ dibutyryl cyclic AMP to the $(+)$ strain, growth did not restart within I h. Similar results were obtained with $(-) M$. hiemalis but during the quiescent period very slow apical growth continued; compare the greater tolerance to dibutyryl cyclic AMP noted above.

\section{Effect of dibutyryl cyclic AMP on mating of $M$. mucedo}

The results (Fig. 9) show that I $\mu$ mol dibutyryl cyclic AMP (in a well) completely inhibits the production of zygospores within $7 \cdot 2 \mathrm{~mm}$ of the well by $56 \mathrm{~h}$. Some zygophores had been present when dibutyryl cyclic AMP was added and some new zygophores appeared during the first $24 \mathrm{~h}$, beyond a zone of 4.5 to $5.8 \mathrm{~mm}$ from the well. These zygophores did not conjugate; those closest to the well aborted, while further away there were some very thin and branched zygophores, often full of carotene, and some appearing quite red. Some 
progametangia had also been present close to the well when the dibutyryl cyclic AMP was added, but these aborted; those further away had a normal appearance but they did not develop into zygospores. Asexual reproduction was less affected, and some $(+)$ sporangiophores were present close to the well.

\section{DISCUSSION}

Some of the species of Mucorales tested (namely B. trispora, P. blakesleeanus and $Z$. moelleri) were not visibly sensitive to dibutyryl cyclic AMP, but $M$. hiemalis and $M$. mucedo underwent profound changes in morphology. The effects in these cultures were initiated swiftly and at low levels of the nucleotide. The abnormalities were clearly due to dibutyryl cyclic AMP and not to osmotic effects such as those observed by Robertson (1958, 1959), as testified by control experiments with $2 \%$ malt. Neither the reasons for this difference in susceptibility nor its significance are clear, especially since Tu \& Malhotra (1977) reported hyphal enlargement, increased frequency of branching and reduced growth rate in $(-) P$. blakesleeanus NRRLI555 exposed to $40 \mu \mathrm{M}$-cyclic AMP. In our experiments $P$. blakesleeanus was exposed to $8 \mathrm{mM}$-dibutyryl cyclic AMP, diluted by diffusion through the agar, without apparent effect. Different species and different isolates may well differ in permeability, in metabolism of dibutyryl cyclic AMP, and in phosphodiesterase levels, and the actual intracellular concentration of the nucleotide may be critical, as there is some evidence for biphasic action in Neurospora crassa (Turian \& Khandjian, 1973).

Organized wall morphogenesis is fundamental to both growth and differentiation in fungi, and among the consequences of adding dibutyryl cyclic AMP to Mucor the effects on wall morphology are conspicuous. Typically, hyphal growth depends on 'apical dominance' or 'polarity', since new wall precursor material is selectively deposited at the hyphal tip for apical extension. Bartnicki-Garcia (1973) has pointed out that this requires a critical enzymic balance between wall softening and wall synthesis. We suggest that dibutyryl cyclic AMP, directly or indirectly, upsets this delicate balance and causes a loss of 'apical dominance'. Apical extension is later re-established, frequently after multiple simultaneous branching, but each modified apex grows at a slower rate than the original single apex. There is no means of telling from these experiments whether the collective growth rate of the new apices was equal to that of the original single apex.

The formation of septa is a particularly striking effect of dibutyryl cyclic AMP since such structures are exceptional in the apical regions of $M$. hiemalis (Fiddy \& Trinci, 1977). Septation and multiple branching occur in response to rather low dibutyryl cyclic AMP levels since they were observed on the edges of the affected zones whilst hyphal tip swelling was not. In the sub-apical region the affected hyphae are visibly thicker than usual; Tu \& Malhotra (1977) showed that walls of $P$. blakesleeanus were up to five times thicker than controls when grown in the presence of $40 \mu \mathrm{M}$-cyclic AMP, and they attributed this effect to increased chitin synthesis at the expense of glycogen.

Some of the strings of septate cells (Fig. 3) superficially resemble the yeast forms of dimorphic Mucorales, in which the prevailing growth form can be determined by environmental conditions. Thus, the yeast form of Mucor racemosus prevails under anaerobic conditions whilst aerobic conditions favour hyphal development. In this species it was reported that the change from yeast to mycelial form on shifting from anaerobic to aerobic conditions was accompanied by a fourfold decrease in endogenous cyclic AMP and could be prevented by additions of dibutyryl cyclic AMP (Larsen \& Sypherd, I 974). However, the treated 'yeast' cells developed atypically into enlarged forms with a conspicuously granular and pigmented cytoplasm.

We suggest that the addition of dibutyryl cyclic AMP to susceptible Mucor hyphae causes a halt in vegetative growth and concomitantly an uncoordinated switch to a number of 'non-growth' activities. Some of these activities mimic certain aspects of sexual and asexual differentiation, for example the formation of septa which normally only delimit 
specialized cells, the swelling following cessation of apical extension which is a characteristic of zygospore suspensors, and the increased production of carotenoids which is a general response to nutritional restrictions and also a specific feature of sexual differentiation. Some of the effects, notably that on apical extension, are immediate and their mechanism must be quite direct, but from the results obtained in the presence of cycloheximide it also appears that some of the effects are relatively indirect, since they also involve the redirection of protein synthesis. The direct observation of these effects in localized areas of established cultures provides a useful counterpart to the data on mass cultures which can be obtained by biochemical means. Together they suggest that localized variations in the level of endogenous cyclic AMP may play an important role in the mediation of externally determined differentiation processes.

We wish to thank Dr G. W. Gooday for introducing us to the polypropylene film technique and for useful discussions.

\section{REFERENCES}

BARTNICKI-GARCIA, S. (1973). Fundamental aspects of hyphal morphogenesis. Symposia of the Society for General Microbiology 23, 245-267.

Bu'Lock, J. D. (1976). Cascade expression of the mating-type-locus in Mucorales. In Proceedings of the 2nd International Symposium on the Genetics of Industrial Microorganisms (1974), pp. 497-509. Edited by K. D. Macdonald. London: Academic Press.

Bu'Lock, J. D. \& Winstanley, D. J. (I97I). Trisporic acid production by Blakeslea trispora and its promotion by barbiturate. Journal of General Microbiology 69, 39I-394.

Bu'Lock, J. D., Jones, B. E. \& Winskinl, N. (1976). The apocarotenoid system of sex hormones and prohormones in Mucorales. Pure and Applied Chemistry 47, 191-202.

CoHen, R. J. (1974). Cyclic AMP levels in Phycomyces in response to light. Nature, London 25I, I44-I46.

Fiddy, C. \& TrincI, A. P. J. (1977). Septation in mycelia of Mucor hiemalis and Mucor rammanianus. Transactions of the British Mycological Society 68, I I 8-120.

GAUGeR, W. L. (1966). Sexuality in azygosporic strains of Mucor hiemalis. I. Breakdown of the azygosporic component. American Journal of Botany 53, 75I-755.

GaUger, W. L. (I975). Further studies on sexuality in azygosporic strains of Mucor hiemalis. Transactions of the British Mycological Society 64, II3-II8.
Gooday, G. W. (1973). Differentiation in the Mucorales. Symposia of the Society for General Microbiology 23, 259-29I.

LARSEN, A. \& SYPHERD, P. S. (1974). Cyclic adenosine $3^{\prime}, 5^{\prime}$-monophosphate and morphogenesis in Mucor racemosus. Journal of Bacteriology II7, 432-44I.

OnIONS, A. H. S. (I97I). Preservation of fungi. Methods in Microbiology 4, I13-15I.

ROBERTSON, N. F. (1958). Observations on the effect of water on the hyphal apices of Fusarium oxysporum. Annals of Botany N.S. 22, 159-I73.

RoBERTSON, N. F. (1959). Experimental control of hyphal branching and branch form in hyphomycetous fungi. Journal of the Linnean Society 56, 207-2II.

Tsuboi, M. \& Yanagishima, N. (1973). Effect of cyclic AMP, theophylline and caffeine on glucose repression of sporulation in Saccharomyces cerevisiae. Archiv für Mikrobiologie 93, I-12.

Tu, J. C. \& Malhotra, S. K. (I977). The significance of cAMP induced alterations in the cellular structure of Phycomyces. Canadian Journal of Microbiology 23, 378-388.

TURIAN, G. \& KHANDIIAN, E. (I973). Action biphasique de l'AMP-cyclique sur la conidiogenèse de Neurospora et la caroténogenèse en général. Berichte der Schweizerischen botanischen Gesellschaft 83, 345-350. 